\title{
TBM
}

\section{Internet-based incentives increase blood glucose testing with a non-adherent, diverse sample of teens with type 1 diabetes mellitus: a randomized controlled Trial}

\author{
Bethany R. Raiff, PhD, ${ }^{1}$ Victoria B. Barrry, PsyD, ${ }^{2,5}$ Ty A. Ridenour, PhD, ${ }^{3}$ Natinee Jitnarin, $P h{ }^{4}$
}

${ }^{1}$ Rowan University, Glassboro, NJ,

${ }^{2}$ Center for Technology and Health, National Development and Research Institutes, Inc., New York, NY 10010, USA

${ }^{3}$ Research Triangle Institutes, Durham NC 12194 UISA

${ }^{4}$ Institute for Biobehavioral Health Research, National Development and Research Institutes, Inc., Leawood, KS 66224 USA

${ }^{5}$ Department of Pediatrics/ Family Care Center,

Harlem Hospital Center, New York NY 10037 USA

Correspondence to: B Raiff raiff@rowan.edu

Cite this as: TBM 2016;6:179-188 doi: 10.1007/s13142-016-0397-5

\section{Abstract}

Non-adherence with self-monitoring blood glucose (SMBG) among teenagers with type 1 diabetes can be a problem. The purpose of this study was to investigate the feasibility, acceptability, and preliminary efficacy of using Internet-based incentives to improve adherence with SMBG in non-adherent teenagers. Participants were randomly assigned to contingent (CS; $N=23$ ), where they had to meet web camera-verified SMBG goals to earn incentives, or non-contingent (NS) groups $(N=18)$, where they earned incentives independent of adherence. Brief motivational interviewing (MI) was given prior to the intervention. Attrition was $15 \%$ in the CS group. Participants and parents endorsed the intervention on all intervention dimensions. Daily SMBG increased after one MI session, and further increased when incentives were added, but significantly more for so for older participants. SMBG declined slowly over time, but only returned to baseline levels for younger NS participants. Internetbased incentive interventions are feasible, acceptable, and show promise for improving adherence with SMBG.

\section{Keywords}

Type 1 diabetes mellitus, Internet, Contingency management, SMBG, Adherence, Incentives

\section{INTRODUCTION}

Despite recommendations to self-monitor blood glucose (SMBG) at least four times per day, adherence among adolescents with type 1 diabetes mellitus (T1D) can be low [1, 2]. Adherence and glycemic control has been found to decline as individuals with T1D transition from childhood to adolescence and adulthood [1, 3-5]. Many factors contribute to these declines, such as reduced parental involvement [1] and environmental or psychosocial barriers [2, 3]. Because of the long-term health benefits of glycemic control, developing a routine of adherence during adolescence is of considerable short- and long-term public health significance [6].

Incentives, such as money or privileges, have been shown to improve adherence to a number of health regimens [7]. This procedure, known as "contingency management" (CM), has been used to treat substance abuse [8], increase asthma and HIV medication

\section{Implications}

Practice: Technology-delivered incentives can be used to increase adherence with blood glucose monitoring in teenagers with type 1 diabetes.

Policy: Policymakers who want to decrease the incidence of diabetes-related health complications should explore sustainable incentive-based interventions to increase adherence with medical regimens that could be implemented wide scale.

Research: Future research should be aimed at identifying procedures for delivering incentivebased interventions that can be sustained and maintained for long periods of time.

adherence [9-11], increase physical activity levels and facilitate weight loss [12, 13], and improve diabetes self-management in children and adolescents [1416]. Contingency management has also been shown to be more effective when combined with other psychosocial interventions, such as motivational interviewing (MI) [17].

The use of Internet-based tools can increase the accessibility of effective, evidence-based interventions such as CM, especially given that in 2008 (close to when the current trial began) approximately $94 \%$ of teenagers reported using the Internet, (96\% White, $92 \%$ African-American, $87 \%$ Hispanic) [18]. Indeed, Internet use, and information technology in general, to improve diabetes management has become increasingly popular [19]. Previous pilot research suggests that internet-based $\mathrm{CM}$ can increase adherence to SMBG among non-adherent teens with T1D on a short-term basis, with teens exhibiting a threefold increase in the number of blood glucose tests taken per day [14].

What remains unknown is whether Internet-based CM can be successfully delivered over longer periods of time with sustained efficacy, as it has been done with other target populations (e.g., drug users, [19]), and whether the improved adherence achieved with Internet-based $\mathrm{CM}$ persists in the post-intervention 
period. To fill these gaps in knowledge, we conducted a small randomized controlled trial to determine the feasibility, acceptability, and preliminary efficacy of an Internet-based CM intervention to improve adherence to SMBG, combined with brief $\mathrm{MI}$, for monitoring and incentivizing adherence among an ethnically, racially, and economically diverse group of nonadherent adolescents diagnosed with T1D $(N=41)$. Additionally, because glycemic control has been shown to be better among younger versus older adolescents (13-15 versus 16-18 years) [20, 21], age was examined as a possible moderator for treatment outcomes. In the current study, feasibility of the intervention was based on willingness to participate (recruitment), adherence with the video submission protocol, and attrition. Acceptability was determined by having participants and their parents complete a Treatment Acceptability Questionnaire at the end of the study, rating the program using $100 \mathrm{~mm}$ visual analog scales (VAS; $1=$ not favorable, $100=$ highly favorable). Finally, preliminary efficacy was based on changes in the frequency of daily SMBG between groups of teenagers receiving contingent incentives (experimental) compared to those receiving non-contingent incentives (control).

\section{Subjects}

Participants $(N=41)$ were adolescents with T1D recruited through an urban outpatient diabetes center. Inclusion Criteria: all participants had to be between the ages of 13-18, diagnosed with T1D for at least one year, report $\mathrm{SMBG}<4$ times per day, live in a home with Internet access, have no travel plans that would inhibit Internet use for 2 months, and read and speak English. Exclusion Criteria: participants could not be within 1 year of their T1D diagnosis due to partial remission that can occur during the first year, and they did not qualify if they had not attended the diabetes clinic within 3 months of the screening. Participants under 18 completed assent and parental/guardian consent. Parents who only spoke Spanish were given translated consent and assessment forms. Institutional review boards for both the research institute conducting the study and the hospital at which the study was conducted approved all procedures.

\section{MATERIALS AND METHODS}

\section{Materials}

Glucometer and testing supplies-Participants were given a Bayer Contour USB ${ }^{\mathrm{TM}}$ glucometer, 200 Contour $^{\mathrm{TM}}$ test strips, and 100 lancets. The Bayer Contour $\mathrm{USB}^{\mathrm{TM}}$ meter connected directly to a computer USB port to generate reports. Additional supplies were provided as needed. Participants were also given a web camera (Creative Labs 1.3 MP Webcam, VF0415) and/or laptop computer (ASUS EeePC) to borrow if needed.

Self-report assessments-During baseline, participants completed: (1) a modified version of the 13-item self- monitoring blood glucose subscale of the Environmental Barriers to Adherence [22] used to identify the participant's perceived environmental barriers to blood glucose testing and (2) a Psychosocial and Medical History Questionnaire, developed for this study to collect demographic information, as well as information about the participant's diabetes and any related medical complications. Exit Session assessments included the following: (1) the Treatment Acceptability Questionnaires (TAQ), on which participants were asked to rate how easy to use, helpful, effective, and fair they viewed the intervention, as well as how much they liked using the study meter and earning incentives, and parents were asked to rate how helpful, enjoyable, and convenient they viewed the intervention, as well as how independent their children were before and during the intervention (using a100-mm visual analog scale). Additional open-ended questions were presented at the end of the survey to identify what they liked about the intervention and what they thought could be done to improve it. (2) A brief medical history assessment which evaluated T1D complications since enrolling. During the followup, the brief medical history assessment was readministered to determine if new complications had arisen.

\section{Procedure}

Screening-The screening took place at a comprehensive care diabetes clinic, comprised of two large waiting rooms, as well as a number of small private exam rooms. Patients were approached while waiting to see a diabetes educator, endocrinologist, psychologist, ophthalmologist, dietician, or social worker as part of routine diabetes care. Either research or clinical staff approached and screened adolescents as they waited for their appointments, and those who met the criteria were invited to participate. Participants completed the informed consent procedure and were then randomly assigned to one of two groups (contingent submission or non-contingent submission), stratifying group assignment based on age (13-15 vs $16-18$ and sex) because of the possibility that age would moderate adherence outcomes. Because of the yoking procedure, the first five participants were automatically assigned to the CS group, which is a common practice when yoking procedures are used in the context of a randomized trial [23-25]. Randomization was determined by entering the participant's ID, age, and sex into a computer program using Excel macros. The macro coded the incoming participant based on both sex and age group and assigned the participant to the group with a lower number of participants with that combined age and sex code. If sex and age distributions were equal across both groups, then the participant was randomly assigned to a group. Finally, if there were an equal number of participants in the two groups then the participant was assigned to the CS group due to the yoking procedure. 
Intake-Next, participants were given equipment, instructed about the study, and administered assessments. Frequency of SMBG was taken from participant's personal use meter or insulin pump for the 5 days preceding intake (pre-MI), and participants were taught how to use the Contour USB ${ }^{\mathrm{TM}}$ as well as the study website (Mōtiv ${ }^{8}$, described below). Participants were given materials to guide them through the website and SMBG recording procedures. Teens then completed a quiz demonstrating that they read and understood the instructions for their group. If they answered a question incorrectly, study staff explained why they had gotten the question wrong and did not proceed until the participant demonstrated that they understood the procedures. Participants received one brief motivational interviewing (MI) session that took place at the end of the Intake. MI sessions lasted approximately $20 \mathrm{~min}$ and were administered by a clinician or research assistant trained by a certified MI instructor.

General procedure-The study was divided into three phases: baseline (5 days), treatment (20 days), and follow-up (20 days).

Baseline-For the first 5 days, participants were told to conduct at least four SMBG per day with their study meter. Participants could record a separate video for each test throughout the day or post one video at the end of the day by scrolling through the log on the meter to display tests taken that day. Participants were told that they would not earn vouchers, which were monetary statements of earnings, during this phase.

Contingent submission treatment condition-After baseline, participants in the contingent submission (CS) group were told that to earn vouchers they needed to submit SMBG videos that showed them meeting their daily goals. The first 3 days served as a shaping period where the goals were gradually increased, starting with a goal of at least one sample on the first day, then two on the second day, and three on the third day. From the fourth day on, participants were required to submit at least four SMBG during a 24-h period (12:00 am11:59 pm) to earn vouchers, separated by at least $2 \mathrm{~h}$ per test. Each test resulted in an immediate $\$ 1.00$ voucher (visible on the study website, Fig. 1a) and they could earn vouchers for up to eight tests per day, as long as the tests were separated by at least $2 \mathrm{~h}$ (as noted above). A $\$ 3.00$ bonus was delivered for the fourth video of the day. CS participants could earn up to $\$ 11.00$ per day, for total possible earnings of $\$ 220$. If participants did not meet the goal, voucher earnings that had been delivered for that day were deducted from their account before they would have the opportunity to spend them (e.g., if three tests were submitted when the goal was four, then $\$ 3.00$ that was initially added was deducted at midnight). Participants were told that they could exchange their vouchers at any time for gift cards at preferred vendors (e.g., Game Zone, Best Buy).

Non-contingent submission treatment condition-Each non-contingent submission (NS) participant was yoked to a participant from the CS group, and the daily earnings for the NS participant were determined by the CS participant to whom they were matched. NS participants were still given the goal of performing and recording at least four SMBG per day (usual care recommendations); however, they were informed that they would be receiving vouchers on a random schedule out of their control and could earn from $\$ 0-\$ 11$ per day. The NS group was not informed of their group assignment until debriefing. The NS group was included to rule out changes in SMBG due to reactivity, access to resources such as incentives, or because of the novelty of the equipment. Yoking is a standard control group in CM interventions to hold the value of earned incentives constant across groups [23-25].

Exit session and follow-up-Exit sessions were conducted at the end of the treatment phase. Participants and their parents completed self-report assessments, and SMBG data were downloaded from the meter. If the participant reported using an alternate meter in addition to the study meter, research staff recorded these results manually. For the follow-up period, participants were asked to continue to use the study meter for 20 days following treatment. During this time, they did not access the website, provide videos, or earn incentives. At the end of the follow-up, personnel conducted a session during which they debriefed participants regarding group assignment. Participants were compensated $\$ 10$ for each of the exit and follow-up sessions.

Mōtiv ${ }^{\circ}-\mathrm{A}$ software engineering company (Red 5 Group) developed a website called Mōtiv ${ }^{8}$ for managing CM programs and collecting SMBG video samples. Study participants logged into Mōtiv ${ }^{8}$ using a unique username and password and completed the SMBG video by following simple on-screen and printed instructions (see Fig. 1). Figure 1a shows the participant's homepage, accessed over a secure, encrypted connection.

To submit a sample, participants clicked the "Post Video" button on the homepage. The video post interface guided the participant through uploading a video sample and posting a result. The participant was instructed to turn on the web camera, click "Record" to leave a sample, and click "Stop" when finished (Fig. 1b). The video stream from the web camera was sent directly to the server over a secure, encrypted connection so that it could not be tampered with by participants.

A properly recorded video contained the participant performing a complete blood glucose test and/or showing past meter result $(\mathrm{s})$ to the camera from the meter log. Participants then entered the numerical result on the meter and posted it in the result text box along with the time when the sample was taken. The result was processed by the Mōtiv ${ }^{8}$ tracking system, with the earnings displayed to participants in the CS group and added to their account history. NS participants automatically received earnings based on their matched CS counterpart at midnight. If participants in either group submitted a sample within the target range (i.e., $70-150 \mathrm{mg} / \mathrm{dl}$ ) they received a 
$\mathrm{A}_{\text {. }}$
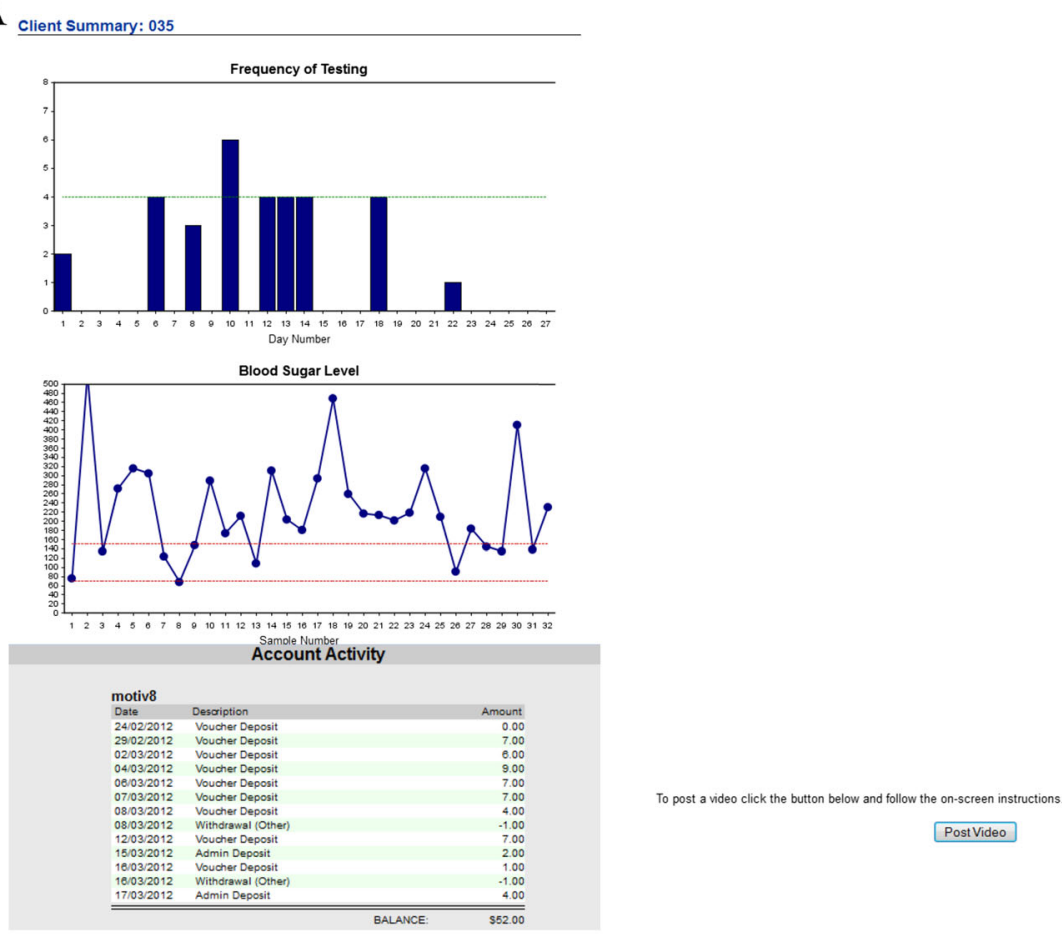

PosiVdeo

$\mathrm{B}$

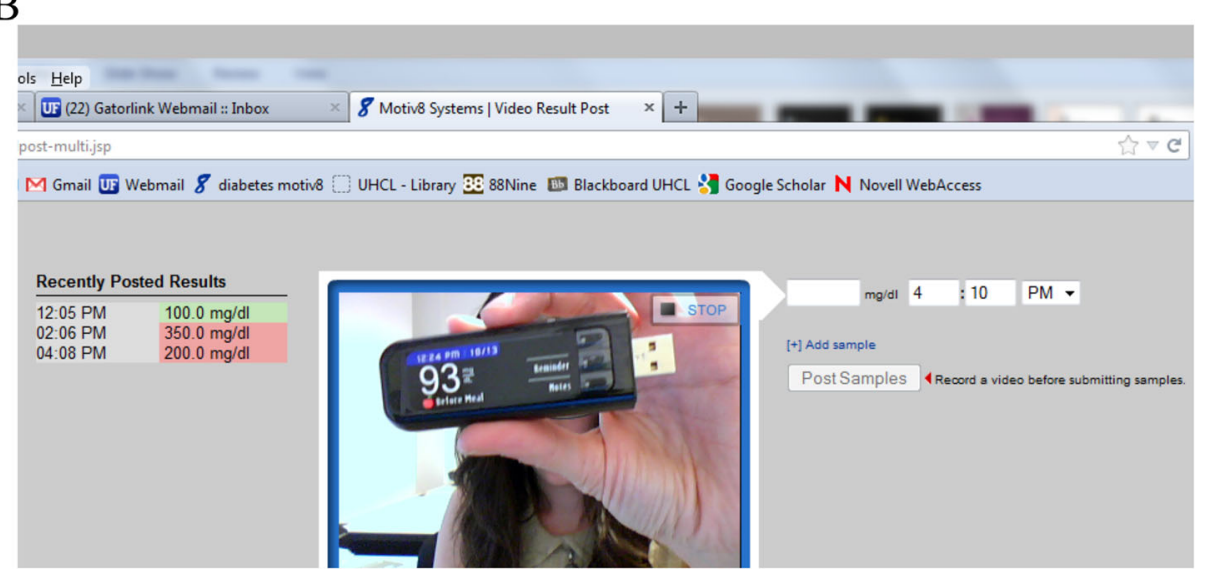

Fig. 1| Screenshots of Mōtiv ${ }^{8}$, the site used to collect blood glucose videos and automatically deliver monetary incentives. a Shows the participant's homepage, which included two graphs (one showing frequency of testing and the other showing blood glucose values), and account earnings. b Shows the screen used to capture videos and manually enter blood glucose values

congratulatory message. If one or more samples were outside of the range, they were told to follow the plan given to them by their diabetes team and to contact the clinic if they needed further assistance. The average time to upload a video after accessing the Mōtiv ${ }^{8}$ website was a couple of minutes, depending on network connectivity. Videos were reviewed by staff daily and the results entered by participants were compared to the results shown on the videos. Any discrepancies were reconciled and vouchers were either approved or rescinded based on the results from videos.

\section{Statistical analyses}

We conducted a power analysis and determined that to detect an $85 \%$ within-subject improvement in adherence from baseline to treatment, a minimum of four participants would be needed per group, whereas to detect a $20 \%$ between-subject improvement, a minimum of 60 participants would be needed per group. This assumes an estimated power of 0.80 and an alpha of 0.05 . The current procedure was expected to produce larger effects than previous research because participants were admitted if they had difficulty adhering and because notification of earned incentives were delivered immediately in the current study, rather than delayed by several days or weeks as in previous studies [16]. For these reasons, and because both within- and between-subject effects were investigated in the current study, we aimed for a sample size of approximately 30 participants per group.

Participants' demographic variables were analyzed using SPSS version 21 (IBM Corp. Released 2012. 
IBM SPSS Statistics for Windows, Version 21.0. Armonk, NY: IBM Corp.). Primary outcomes included the daily frequency of SMBG. Secondary outcomes included feasibility, as measured by willingness to participate (recruitment), adherence with the video submission protocol, and attrition. Acceptability was determined by answers on the Treatment Acceptability Questionnaire at the end of the study (VAS; $1=$ not favorable, $100=$ highly favorable). Finally, mean blood glucose (BG) levels across phases, between groups, was calculated.

Student's $t$ tests were used to determine whether there were significant differences between groups on continuous variables, and chi-square tests were used for categorical variables. For non-normal continuous variables, the non-parametric MannWhitney $U$ test was conducted. A repeated measures mixed analysis of variance (ANOVA) was used to determine whether there were differences in BG levels between groups across study phase. Significant differences were based on $\alpha$ set to $p<0.05$. Mixed model trajectory analysis (MMTA) [26-28] was used (in SAS 9.3) to test changes in daily SMBG associated with each intervention component with regard to mean change and slope over time. Additional planned analyses were conducted to determine whether age (13-15 vs 16-18) served as a moderator to treatment effects, based on previous research, suggesting that age is related to metabolic control [20,21]; thus, age was tested as a dichotomized variable $(0=$ younger, $1=$ older $)$. Each intervention component was tested as a fixed effect for a mean and a slope effect. To account for autocorrelation, a Toeplitz error covariance structure best fit the data compared to variance components, unstructured, autocorrelation (lag 1), and compound symmetry. Missing data were analyzed as missing and were not significantly different between groups when excluding non-responders $(\mathrm{CS}=5 \%, \mathrm{NS}=5.7 \%$, overall= $5.3 \%)$. The Kenward-Roger adjustment [27] was used in all MMTA.

\section{Results}

Feasibility

Recruitment took place from April 2011 through November 2012. Adolescents $(N=274)$ were approached to determine whether they were interested and qualified for the study, of which 68 qualified and consented to participate. Fifty-two of those who consented, enrolled in the study $(76 \%)$. The primary reason individuals did not qualify was because they reported already testing their blood glucose four or more times per day $(n=125,43 \%)$. Additional reasons included not having a T1D diagnosis, reporting a disability that interfered with the study, not speaking English, or being ineligible for some other reason $(n=60 ; 20 \%)$. Only seven teens did not have Internet access (2\%) and only 19 teens who were approached did not enroll due to lack of interest $(8 \%)$. See Fig. 2 for the
Consolidated Standards of Reporting Trials (CONSORT) flow diagram.

After consenting and randomization, 11 (22\%) participants $(\mathrm{CS}=4, \mathrm{NS}=7)$ were considered "nonresponders" and were excluded from analyses for failing to provide SMBG records on $50 \%$ or more of study days (via Mōtiv ${ }^{8}$ and/or meter log). Nineteen $(83 \%)$ responders in the CS group, and $18(100 \%)$ in the NS group, submitted at least one video sample via the Mótiv ${ }^{8}$ website. Overall, responders submitted videos an average of 19 (S.D. \pm 7.26 ) out of the 25 study days.

Demographic and diabetes characteristics of responders $(\mathrm{CS}=23, \mathrm{NS}=18)$ are presented in Table 1 . There were no statistically significant differences in demographic and diabetes characteristics among NS and CS groups. Participants were predominantly male minorities (Hispanic or African-American). About half of participants were living in a household with an annual income of $\$ 45,000$ or less. Mean participant age was $15.20( \pm 1.43)$ and mean age of diagnosis was $7.89( \pm 3.96)$. Average $\mathrm{HbA}_{1 \mathrm{C}}$ at intake was 9.60 $( \pm 1.71)$. The average amount of monetary incentives earned was similar across groups $(M=\$ 64.77$, range \$0-162).

\section{Acceptability}

Figure 3 shows the mean and individual acceptability rating from the TAQ for both the teenagers and their parents across the CS and NS groups. No significant differences were found between groups for mean VAS ratings $(0=$ not favorable, $100=$ very favorable $)$ regarding how easy to use (Mdn; IQR $=80.00$; 36.25), helpful (Mdn; IQR = 75.50; 41.25), convenient (Mdn; $\mathrm{IQR}=69.00 ; 39.5)$, and effective $(\mathrm{Mdn} ; \mathrm{IQR}=66.50$; 41.50) they viewed the Mōtiv ${ }^{8}$ system. Both groups liked the study glucometer (Mdn; IQR $=89.50 ; 29.5$ ) and earning monetary vouchers $(\mathrm{Mdn} ; \mathrm{IQR}=90.5$; 21.75). There was a significant difference with how fair the participants viewed the intervention, with NS participants rating the intervention as being more fair than CS participants (CS: Mdn; IQR $=87.00 ; 27.00$ vs NS: $\mathrm{Mdn} ; \mathrm{IQR}=96.00 ; 12.00, U=119, \mathrm{p}=0.034)$.

There were also no significant differences for the parent TAQs on any of the VAS ratings regarding how helpful (Mdn; IQR $=80.5 ; 28.5)$, enjoyable $(\mathrm{Mdn} ; \mathrm{IQR}=83.5 ; 40.0)$, or convenient (Mdn; $\mathrm{IQR}=84.0 ; 28.75)$ they viewed Mōtiv ${ }^{8}$ for their child. There were also no significant group differences between ratings of how independent their child was with diabetes management before versus during treatment $(\mathrm{Mdn}$; IQR Before $=83.50 ; 40.00$ versus during $=84.00 ; 28.75$ ).

Open-ended questions on the TAQ were assessed for common themes by two independent raters. Almost $90 \%$ of participants in both groups reported that there were times when they tested their blood glucose but did not submit a video on the website, the primary reason was not having access to their home computer or Internet $(51 \%)$. Most participants felt the program

page 183 of 188 


\section{CONSORT Flow Diagram}

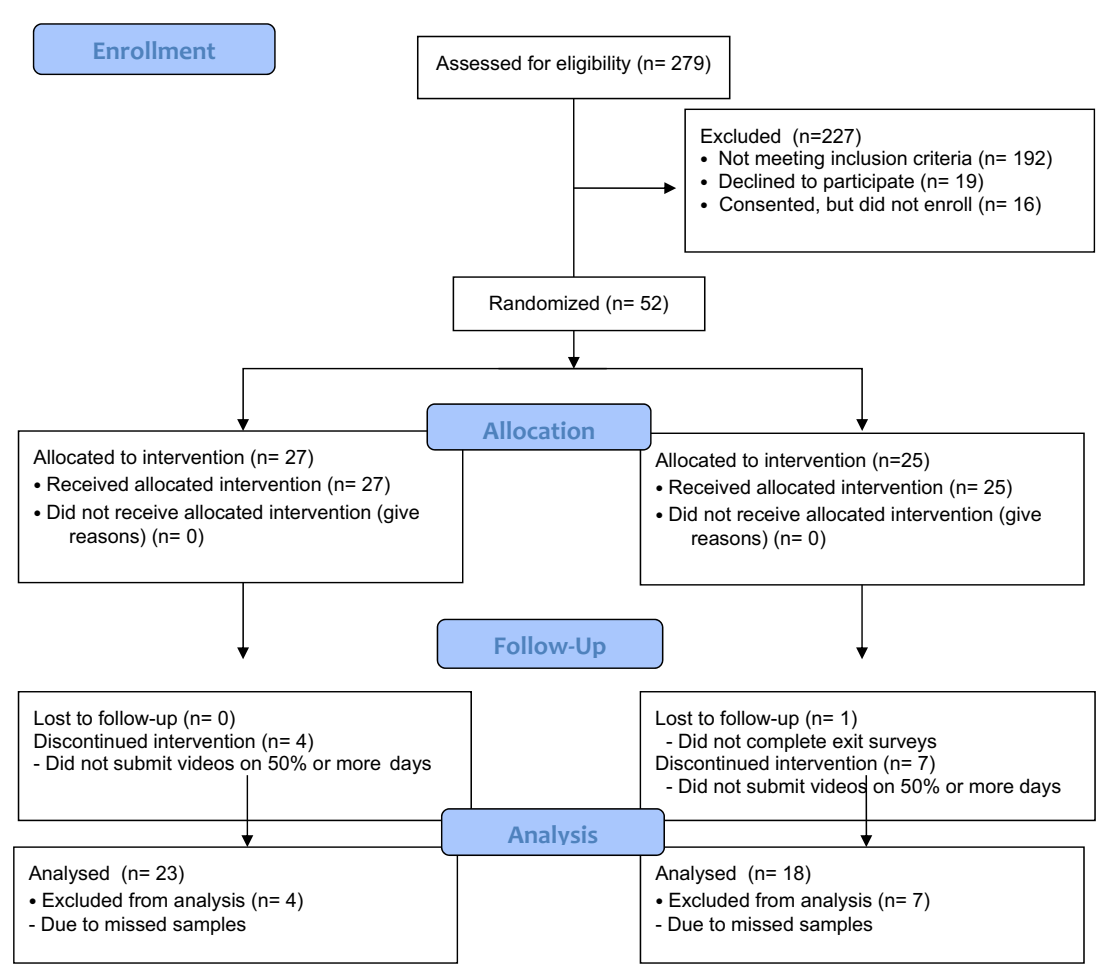

Fig. 2 | CONSORT Flow Diagram outlines the number of participants who were involved at each stage of the study

would be more convenient by mobile phone $(66 \%)$. The most noted positive aspect was that it helped them track their blood glucose and time of testing $(29 \%)$, followed by the ability to earn incentives for testing (24\%). Thirty-nine percent of participants did not give a response to what they liked least about the program. The most common suggestion parents gave for improving the program was to incorporate social support, such as an online chat room or in-person support for teens with T1D.

\section{Preliminary efficacy}

Because the goals of this study were to evaluate the preliminary efficacy of the intervention, an intent-totreat analysis was not used. Figure 4 shows the mean $( \pm \mathrm{SEM})$ frequency of SMBG per day across conditions for both groups. The frequency of SMBG increased during baseline and treatment for both groups, and decreased during the follow-up condition.

Table 2 presents improvements in fit to the data that were associated with age group, the baseline (post-MI), Internet monitoring plus treatment (CS or NS), and being in the CS group. Only the factors which significantly improved fit are presented. The comparison model included fixed effects for time as a linear slope (quadratic slope was tested, but $p>0.05$ ), a mean effect of MI (linear slope was tested, but $p>0.05$ ) and fixed and random intercept effects (no random effects for time were statistically significant).
Figure 5 presents the best fitting model as a trajectory and an equation. Baseline was associated with about 1.0 more glucose test per day on average (0.99). On average, an additional 1.3 glucose tests (1.32) were associated with receiving treatment and another two thirds of a test per day was associated with CS group assignment (0.66). Intervention effects gradually declined during the 20-day treatment, but much more for younger teens. Finally, results suggest that older adolescents responded better to having treatment (1.04 more daily glucose tests) than younger adolescents. No age differences were found during baseline or in response to $\mathrm{MI}$, but the age differences that were revealed during the treatment phase did maintain during follow-up. No significant differences were found in the mean BG levels between groups across the four study phases (CS: pre-MI=217.9 \pm 94 , baseline $=250.9 \pm 82$, treatment $=268.1 \pm 72$, followup $=253.6 \pm 87$ versus NS: pre-MI $=220.7 \pm 81$, baseline $=228.1 \pm 69$, treatment $=246.6 \pm 83$, followup $=258.0 \pm 106$.

\section{Discussion}

This study is the first randomized controlled trial investigating the feasibility, acceptability, and preliminary efficacy of Internet-based monitoring and incentivizing of SMBG in teens diagnosed with T1D. The intervention was found to be both feasible and 
Table 1 | Demographic and diabetes characteristics (M [SD] or \%) of participants

\begin{tabular}{|c|c|c|}
\hline Variables & $\begin{array}{l}\text { Non-contingent group } \\
(n=18)\end{array}$ & $\begin{array}{l}\text { Contingent group } \\
(n=23)\end{array}$ \\
\hline \multicolumn{3}{|l|}{ Demographics } \\
\hline Age, years & $15.33 \pm 1.37$ & $15.04 \pm 1.49$ \\
\hline Gender, $\%$ male & 55.6 & 60.9 \\
\hline \multicolumn{3}{|l|}{ Race, \% } \\
\hline White & 23.5 & 21.7 \\
\hline Hispanic & 64.7 & 60.9 \\
\hline African-American & 11.8 & 13.0 \\
\hline Multiracial & 0 & 4.4 \\
\hline \multicolumn{3}{|l|}{ Household income, \% } \\
\hline$<\$ 30 \mathrm{~K}$ & 38.9 & 26.1 \\
\hline$\$ 30 \mathrm{~K}-45 \mathrm{~K}$ & 5.5 & 26.1 \\
\hline$\$ 45 \mathrm{~K}-60 \mathrm{~K}$ & 11.1 & 4.3 \\
\hline$\$ 60 \mathrm{~K}-75 \mathrm{~K}$ & 0 & 8.7 \\
\hline$\$ 75 \mathrm{~K}-100 \mathrm{~K}$ & 5.6 & 4.4 \\
\hline$\$ 100 \mathrm{~K}-150 \mathrm{~K}$ & 22.2 & 13.0 \\
\hline$\$ 150 \mathrm{~K}-250 \mathrm{~K}$ & 5.6 & 13.0 \\
\hline$>\$ 250 \mathrm{~K}$ & 0 & 0 \\
\hline Unknown & 11.1 & 4.4 \\
\hline \multicolumn{3}{|l|}{ Diabetes status } \\
\hline Age at diagnosis, years & $8.31 \pm 4.32$ & $7.30 \pm 3.79$ \\
\hline Intake HbA1C value $(\mathrm{mmol} / \mathrm{mol})$ & $9.44 \pm 1.60$ & $9.75 \pm 1.86$ \\
\hline Vouchers (\$) earned & $\$ 62.65$ (range $\$ 0-162$ ) & $\$ 66.41$ (range $\$ 0-162$ ) \\
\hline
\end{tabular}

acceptable, given the relatively high rate of enrollment among individuals who were approached and qualified for the study (only $8 \%$ declined), the low rate of attrition in the CS group (15\%), and the high, positive endorsement of all aspects of the intervention. Additionally, the study was designed to examine the
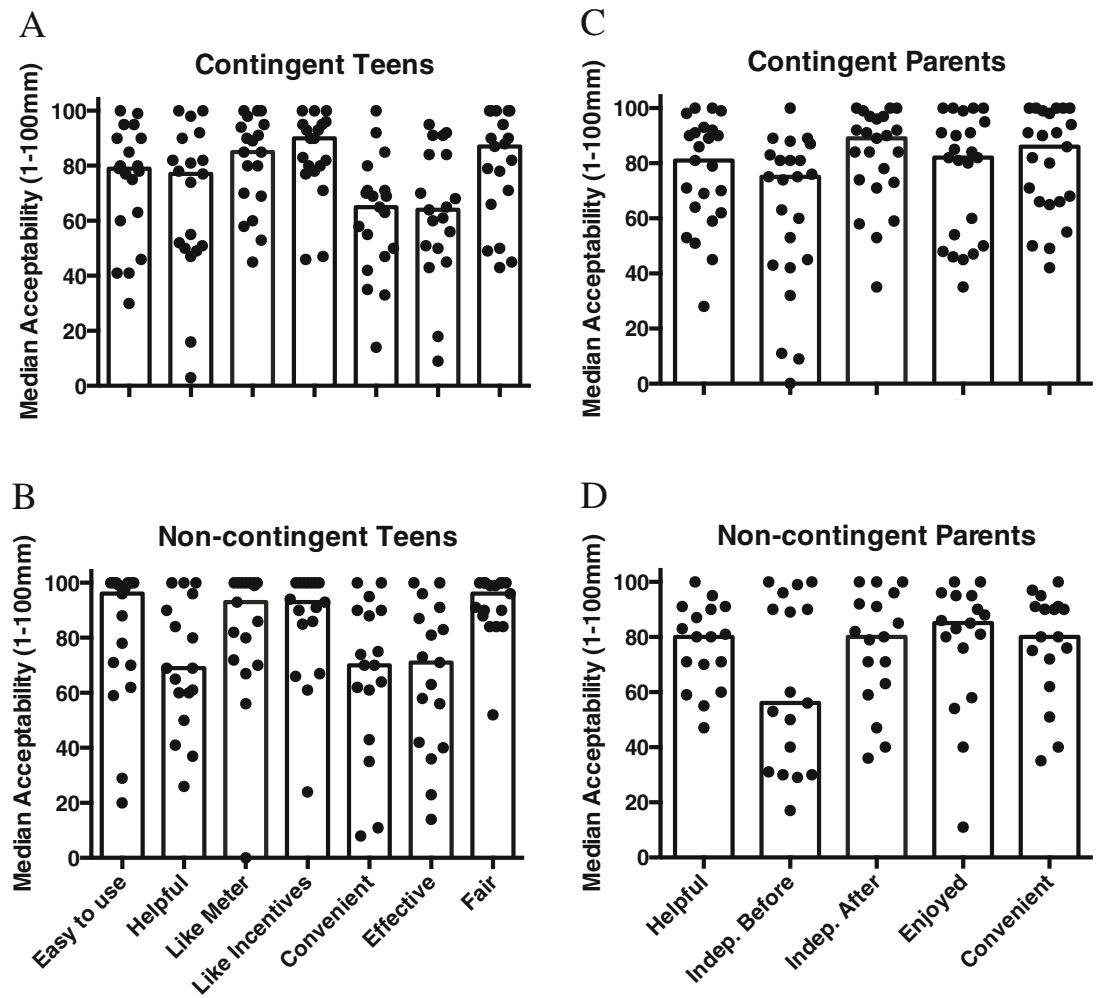

Fig. 3 | Individual (dots) and mean (white bar) scores from the Treatment Acceptability Questionnaire for the contingent (top row) and the non-contingent (bottom row) teenagers and their parents. There were no significant differences between groups on any of these measures 


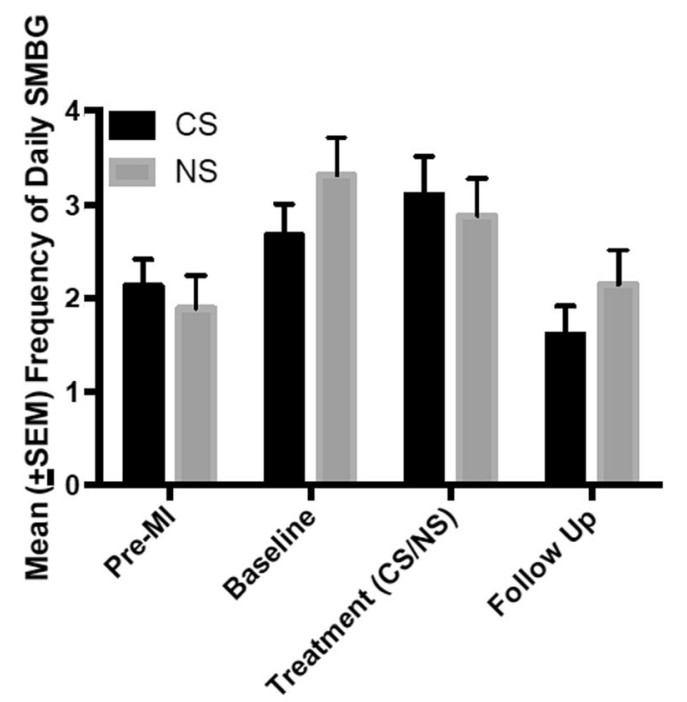

Condition

Fig. 4 | Mean ( \pm SEM) frequency of daily blood glucose monitoring for contingent (black bars) and non-contingent (gray bars) submission participants during each of the study conditions (pre-MI, baseline, treatment, follow-up)

preliminary efficacy of the intervention, by making both within and between group comparisons. The non-contingent group provided information about the incremental impact of adding contingent incentives, but was otherwise an active treatment group (given MI exposure, video monitoring, and interactions with study staff). All participants showed an increase in the frequency of SMBG after receiving one brief MI session ( $20 \mathrm{~min})$, consistent with past research showing the potential benefits of MI [28]. The addition of incentives further increased frequency of testing, but more so for participants in the contingent group, suggesting that making the incentives contingent on objectively verified target behaviors is an important element of an incentive-based intervention, especially with younger teenagers.

The current findings also showed that older participants (16-18 years old) had significantly greater improvements in the frequency of SMBG than younger participants (13-15 years old), regardless of group assignment, and that the increased benefits were more likely to maintain during follow-up. This is surprising given that past research suggests adherence tends to decline as children get older [1, 20,21]. It is possible that monetary incentives were more powerful for older teens, many of whom reported having jobs and were, therefore, more financially independent from their parents.

However, these age differences should be evaluated with caution. Of the 11 excluded participants for being non-responders, eight were between the ages of 1618 years of age. Unfortunately, the current study was not large enough to detect potential differences between responders and non-responders on moderator variables, such as age. Nevertheless, and despite the small sample size, these significant age-related outcomes suggest that it would be worthwhile to investigate further in a larger trial.

A few limitations of the study are worth noting. First, recruitment relied on self-reported non-adherence and took place while patients waited for clinic appointments. It is possible that patients most in need of an intervention were not captured in this study, either because they falsified their self-reported adherence due to social pressure (i.e., parents) or because they were less likely to attend clinic appointments. Missed doctor appointments are associated with poorer glycemic control and non-adherence [29]. Different recruitment strategies may be needed to reach these higher risk patients.

Second, the current study required participants to post videos of their SMBG every day to receive incentives because it is well established that immediate, frequent incentives are more effective at changing behavior than delayed, less frequent incentives [30]. Although participants rated most elements of the intervention favorably, they rated convenience of the intervention lower, and would have preferred it be administered via mobile devices. Smartphones were less prominent when this study began (2010), which is why they were not used. However, recent research suggests that smartphones can be used to effectively deliver incentive-based interventions for diabetes management [30].

Additionally, glucose monitoring technology has changed over the past 5 years, with a number of

\begin{tabular}{|c|c|c|c|c|c|}
\hline Model (Fixed Effects) & $\mathrm{AIC}$ & $\mathrm{BIC}$ & $-2 \mathrm{LL}$ & df & $L R x^{2}, d f^{a}$ \\
\hline 1. Intercept, time, MI & 6957.2 & 6965.6 & 6947.2 & 6 & - \\
\hline 2. Added contingency mean effect & 6780.9 & 6791.0 & 6768.9 & 7 & $178.3,1$ \\
\hline 3. Added contingency slope effect & 6722.7 & 6734.5 & 6708.7 & 8 & $60.2,1$ \\
\hline 4. Added interaction between contingency and age & 6717.4 & 6730.9 & 6701.4 & 9 & $7.3,1$ \\
\hline 5. Added mean effect of CS & 6674.5 & 6707.7 & 6674.5 & 10 & $26.9,1$ \\
\hline $\begin{array}{l}\text { 6. Added 3-way interaction between contingency, } \\
\text { time and age }\end{array}$ & 6689.9 & 6706.8 & 6669.9 & 11 & $4.6,1$ \\
\hline \multicolumn{6}{|c|}{$\begin{array}{l}\text { Kenward-Roger correction was used. The only significant random effect was the intercept. Only the factors that contributed statistically significant improvement t } \\
\text { model fit are presented. AICAikake's Information Criterion, BICBayes Information Criterion, Contingency receiving NS or CS, } L L \log \text { likelihood, } L R \text { likelihood ratio } \\
{ }^{a} \text { for all } L R X^{2} \text { tests, the comparison model is the preceding model and } p<0.01\end{array}$} \\
\hline
\end{tabular}




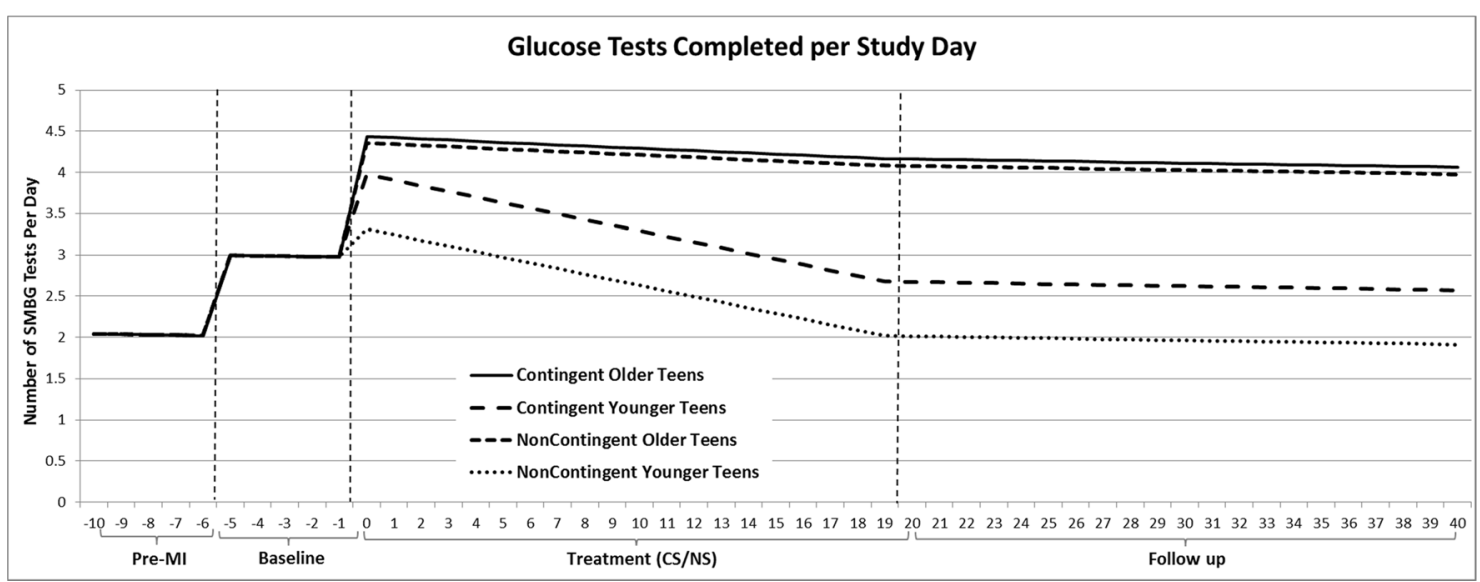

Fig. 5 | The number of tests per day shown as a fitted function from the MMTA analyses for each of the study conditions (pre-MI, baseline, treatment, follow-up). There were no significant differences between groups during pre-MI or baseline, which is indicated by the solid black line. During treatment and follow-up, significant differences across contingent older (solid), contingent younger (large dashes), non-contingent older (medium dashes), and non-contingent younger (small dashes) participants are noted. The corresponding MMTA equation is frequency of tests $=1.9885-0.00501$ (per day) +0.9805 (for Motivational Interviewing) +1.3240 (during treatment phase) -0.06317 (per day of treatment phase) +1.0430 (older teens during treatment phase) +0.6598 (while receiving CS) -0.05378 (per day of treatment phase for younger teens). Each model parameter reached $p<0.01$ statistical significance

meters, continuous glucose monitors, and applications now available that can communicate with smart devices automatically (e.g., Telcare ${ }^{\mathrm{TM}}$, iHealth ${ }^{\circledR}$, Dexcom Share, Medtronic Connect). These technological advances should be harnessed to simplify the remote monitoring and delivery of incentive-based interventions, eliminating the need for video submissions. It is important to note, however, that during the current study, it became clear that participants were being directed by doctors to use multiple meters as a strategy to increase SMBG. This is important for anyone planning to rely on a single meter to monitor adherence with SMBG recommendations.

Third, although there were significant increases in the daily frequency of SMBG, this was the first study of incentive-based interventions for SMBG to use a multi-level modeling trajectory analysis, and in doing so, it was shown that there was a threeway interaction between group assignment, age, and time in the study. Although there were declines in the observed changes in daily SMBG, the declines much more pronounced in younger participants than older participants. These age-related differences in the maintenance of treatment effects is worth investigating further, and it will be especially important to explore methods for increasing maintenance among younger participants. In a small, within-subject pilot study, Cafazzo and colleagues [30] showed that teenagers increased their daily frequency of SMBG when doing so was gamified with points (that could later be used to purchase music and apps from the iTunes store) delivered for daily SMBG, as verified by a smartphone application and a bluetooth glucometer. Participants could also interact with peers via the application, similar to online social networking sites. This gamification approach may be more sustainable, and the social support may facilitate longer-term engagement, as parents in the current study suggested might have improved the Internet-based CM intervention. However, a larger, longer randomized controlled clinical trials involving trajectory analyses, such as the multi-level modeling used in the current study, are needed.

Fourth, although increases in SMBG were observed, the current study did not determine whether these increases were associated with improved glycemic control. There were no differences in BG levels across study conditions. It should be noted, however, that relying on BG alone has limitations due to the low number of SMBG tests during the baseline conditions (because of poor adherence), and also because BG fluctuates throughout the day and can be used to inform corrective actions (i.e., a high number may occur which would allow insulin dose adjustments to take place). Future studies should include follow-up glycated hemoglobin (A1c) tests to determine whether increases in testing are associated with overall changes in glycemic control.

Two additional, promising pilot studies examining incentive-based approaches to sustaining adherence and improving glycemic control were recently published. First, Stanger and colleagues [15] taught parents of teens with T1D to deliver daily incentives or punishers based on SMBG adherence, and parents received rewards for correctly implementing the contract. Teaching parents the skills to deliver incentives contingent on objective evidence of proper diabetes management (e.g., using a wireless meter) may result in longer-term maintenance of treatment effects, especially with younger participants. Second, Petry and colleagues [31] tested a very low cost incentive-based intervention, rewarding teens with $\$ 0.10$ for each test conducted during the day, with bonuses for testing at least four times per day. Adherence increased and was associated with improvements in $\mathrm{HbA}_{1 \mathrm{C}}$ that were 
sustained in the majority of participants for nearly one year after the intervention ended, suggesting incentivebased interventions may be able produce lasting changes in the long-term health of teenagers with T1D. Neither of these studies included control groups; therefore, additional research is needed to further support these promising preliminary results.

In conclusion, as the first randomized controlled trial investigating an Internet-based CM intervention, this study demonstrated the importance of making monetary incentives contingent on SMBG adherence, particularly in older teenagers. Future randomized controlled trials should further explore the increased convenience, acceptability, and sustainability of incorporating technological advances, such as wireless glucometers and gamification, that allow for immediate, frequent, and sustainable monitoring and feedback, of incentive-based interventions.

Compliance with ethical standards Participants under 18 completed assent and parental/guardian consent. Parents who only spoke Spanish were given translated consent and assessment forms. Institutional review boards for both the research institute conducting the study and the hospital at which the study was conducted approved all procedures.

\section{References}

1. Anderson B, Ho J, Brackett J, et al. Parental involvement in diabetes management tasks: relationships to blood glucose monitoring adherence and metabolic control in young adolescents with insulindependent diabetes mellitus. J Pediatr. 1997; 130(2): 257-265.

2. Berg CA, Wiebe DJ, Suchy Y, et al. Individual differences and day-today fluctuations in perceived self-regulation associated with daily adherence in late adolescents with type 1 diabetes. I Pediatr Psychol. 2014; 39(9): 1038-1048.

3. Bryden KS, Dunger DB, Mayou RA, et al. Poor prognosis of young adults with type 1 diabetes: a longitudinal study. Diabetes Care. 2003; 26(4): 1052-1057.

4. Crowley R, Wolfe I, Lock K, et al. Improving the transition between paediatric and adult healthcare: a systematic review. Arch Dis Child. 2011; 96(6): 548-553.

5. Svoren BM, Volkening LK, Butler DA, et al. Temporal trends in the treatment of pediatric type 1 diabetes and impact on acute outcomes. J Pediatr. 2007; 150(3): 279-285.

6. The Diabetes Control and Complications Trial/Epidemiology of Diabetes Interventions and Complications (DCCT/EDIC) Study Research Group. Intensive diabetes treatment and cardiovascular disease in patients with type 1 diabetes. N Engl J Med. 2005; 353(25): 2643-2653.

7. Petry NM, Rash CJ, Byrne S, et al. Financial reinforcers for improving medication adherence: findings from a meta-analysis. Am J Med. 2012; 125(9): 888-896.

8. Higgins ST, Silverman K, Heil SH. Contingency Management in Substance Abuse Treatment. New York: Guilford Press; 2007.

9. Burkhart PV, Rayens MK, Oakley MG, et al. Testing an intervention to promote children's adherence to asthma self-management. J Nurs Scholarsh. 2007; 39(2): 133-140.

10. Rosen MI, Dieckhaus K, McMahon TJ, et al. Improved adherence with contingency management. AIDS Patient Care STDS. 2007; 21(1): 30-40.

11. Sorensen JL, Haug NA, Delucchi KL, et al. Voucher reinforcement improves medication adherence in HIV-positive methadone patients: a randomized trial. Drug Alcohol Depend. 2007; 88(1): 54-63.

12. Andrade LF, Barry D, Litt MD, et al. Maintaining high activity levels in sedentary adults with a reinforcement-thinning schedule. J Appl Behav Anal. 2014; 47(3): 523-536.

13. John LK, Loewenstein G, Troxel AB, et al. Financial incentives for extended weight loss: a randomized, controlled trial. J Gen Intern Med. 2011; 26: 621-626.

14. Raiff B, Dallery J. Internet-based contingency management to improve adherence with blood glucose testing recommendations for teens with type 1 diabetes. J Appl Behav Anal. 2010; 43: 487-491.

15. Stanger C, Ryan SR, Delhey LM, et al. A multicomponent motivational intervention to improve adherence among adolescents with poorly controlled type 1 diabetes: a pilot study. J Pediatr Psychol. 2013; 38(6): 629-637.

16. Wysocki T, Green L, Huxtable K. Blood glucose monitoring by diabetic adolescents: compliance and metabolic control. Health Psychol Off J Div Health Psychol Am Psychol Assoc. 1989; 8(3): 267-284.

17. Rohsenow DJ, Tidey JW, Martin RA, Colby SM, Sirota AD, Swift RM, et al. Contingent vouchers and motivational interviewing for cigarette smokers in residential substance abuse treatment. J Subst Abuse Treat [Internet]. 2015 [cited 2015 Jun 8]; Available from: http:// www.sciencedirect.com/science/article/pii/S074054721500063X

18. Pew Research Center. The lives of teens and their technology [Internet]. 2008. Available from: http://www.pewinternet.org/2008/04/ 24/the-lives-of-teens-and-their-technology/

19. Silverman K, Svikis D, Wong C, et al. A reinforcement-based therapeutic workplace for the treatment of drug abuse: three-year abstinence outcomes. Exp Clin Psychopharmacol. 2002; 10(3): 228-240.

20. Clements MA, Foster NC, Maahs DM, Schatz DA, Olson BA, Tsalikian $\mathrm{E}$, et al. Hemoglobin A1C (HbA1c) changes over time among adolescent and young adult participants in the T1D exchange clinic registry. Pediatr Diabetes. 2015.

21. Gerstl E-M, RabI W, Rosenbauer J, et al. Metabolic control as reflectet by $\mathrm{HbA} 1 \mathrm{c}$ in children, adolescents and young adults with type-1 diabetes mellitus: combined longitudinal analysis including 27,035 patients from 207 centers in Germany and Austria during the last decade. Eur / Pediatr. 2007; 167(4): 447-453.

22. Irvine A, Saunders J, Blank M, et al. Validation of scale measuring environmental barriers to diabetes-regimen adherence. Diabetes Care. 1990; 13(7): 705-711.

23. Higgins S, Wong C, Badger G, et al. Contingent reinforcement increases cocaine abstinence during outpatient treatment and 1 year follow-up. J Consult Clin Psychol. 2000; 68(1): 64-72.

24. Silverman K, Higgins ST, Brooner RK, et al. Sustained cocaine abstinence in methadone maintenance patients through voucher-based reinforcement therapy. Arch Gen Psychiatry. 1996; 53(5): 409-415.

25. Silverman K, Wong C, Umbricht-Schneiter A, et al. Broad beneficial effects of cocaine abstinence reinforcement among methadone patients. J Consult Clin Psychol. 1998; 66(5): 811-824.

26. Ferron JM, Farmer JL, Owens CM. Estimating individual treatment effects from multiple-baseline data: a Monte Carlo study of multilevel-modeling approaches. Behav Res Methods. 2010; 42(4): 930-943.

27. Ridenour TA, Pineo TZ, Molina MMM, et al. Toward rigorous idiographic research in prevention science: comparison between three analytic strategies for testing preventive intervention in very small samples. Prev Sci. 2013; 14(3): 267-278.

28. Kenward MG, Roger JH. Small sample inference for fixed effects from restricted maximum likelihood. Biometrics. 1997; 53(3): 983-997.

29. Karter AJ, Parker MM, Moffet HH, et al. Missed appointments and poor glycemic control: an opportunity to identify high-risk diabetic patients. Med Care. 2004; 42(2): 110-115.

30. Cafazzo JA, Casselman M, Hamming N, Katzman DK, Palmert MR. Design of an mHealth app for the self-management of adolescent type 1 diabetes: a pilot study. J Med Internet Res [Internet]. 2012 May 8 [cited 2015 Jun 1];14(3). Available from: http://www.ncbi. nlm.nih.gov/pmc/articles/PMC3799540/

31. Petry NM, Cengiz E, Wager J, et al. Testing for rewards: a pilot study to improve type 1 diabetes management in adolescents. Diabetes Care. 2015; 38(10): 1952-1954. 